\title{
Failure to find evidence of the unitary OR concept with indifferent low-intensity auditory stimuli
}

\author{
ROBERT J. BARRY \\ School of Education, University of New South Wales, Kensington, 2033, Australia
}

\begin{abstract}
The most commonly discussed indices of the OR (e.g., GSR, respiration, HR, CPV, PPV, and EEG) have been traditionally conceptualized as showing similar behavior in response to stimulus variation. Two of Sokolov's hypothesized dimensions of stimulus variation affecting OR behavior are intensity and novelty, and these were focused upon in the present study. The six psychophysiological indices listed above were found to exhibit markedly different behaviors in their responses to manipulation of these two independent variables. These differences were so marked as to make the concept of a unitary OR untenable.
\end{abstract}

Sokolov's concept of the orienting response (OR) has attracted much attention in western psychophysiology in the last decade because of the order it promised to bring to a fairly chaotic area of stimulusresponse relationships. The $\mathrm{OR}$ was described by Sokolov (1960) as "an unspecific reflex . . . independent of the modality of the stimulating agent"' (p. 189). This nonspecificity was of considerable importance in the elucidation of those physiological responses considered by Sokolov as part of the OR, and which included "changes of respiration, vascular responses, heart rate and the SGR" (GSR) and "EEG components, which include alpha-rhythm depression or arousal"' (Sokolov, 1960, p. 227). These indicators of the OR were claimed by Sokolov to be particularly sensitive to variation in the stimulus dimensions of novelty and intensity. The most striking of these features of the OR was its dependence on the novelty or newness of the eliciting stimulus: "the orienting reflex ... is specifically evoked by the novelty of a stimulus"' (Sokolov, 1963a, p. 547). As the novelty of a stimulus was reduced by repeated presentation, habituation of the OR was claimed: "When the same stimulus is repeatedly applied, orienting reactions to it become extinguished" (Sokolov, 1955, p. 134). The second property of sensitivity to stimulus intensity is not as simply described, since the relationship itself is most complex. Sokolov (1963b), when writing of specific OR indicators, claimed that "In the medium range of stimulus intensity, the magnitude of cephalic and hand vasomotor reaction increases with stimulus intensity"' (p. 51). On p. 180 of the same publication, these measures appear to be linearly related to the intensity of an auditory stimulus over a range from 20 to $60 \mathrm{~dB}$, while the GSR exhibits a similar relationship (p. 63) from 50 to

Address requests for reprints to Robert J. Barry, School of Education, University of New South Wales, Kensington, 2033, Australia.
$90 \mathrm{~dB}$. With very low stimulus intensities, intensification of the OR was said to occur: "the size of the reaction is somewhat greater in the range of weak stimuli than the medium ones, after which it steadily increases with the intensity of stimuli" (Sokolov, $1963 \mathrm{~b}, \mathrm{p} .63)$. The figure accompanying this last statement appears to indicate that the "range of weak stimuli" could extend up to $50 \mathrm{~dB}$ for auditory stimulation. With high intensities of stimulation, the OR is supposedly replaced by a defense reaction (DR), which is beyond the scope of the present discussion.

In relation to these determinants of the $O R$, Sokolov has consistently used the concept of a single response system, describing the $O R$ as "a complex reaction of the whole organism" (1955, p. 134), "a unitary system" (1960, p. 191), "a functional system," "an integrated reaction" (1963a, p. 547), and "an independent functional system" (1963b, p. 12). Such statements have not been explicitly elaborated by Sokolov, but it would appear from his common usage of the term "OR" in the singular sense, together with these phrases, that the various physiological indices mentioned above are conceptualized as behaving in the same way in response to variation in stimulus novelty and intensity.

It is difficult to see how such a unitary OR concept can be held by Sokolov when a report by Voronin and Sokolov (1960) is considered. In this study, 100 subjects were stimulated with a $1,000-\mathrm{Hz} 50-\mathrm{dB}$ tone and the occurrence of OR indicators noted. Occipital alpha blocking occurred in 85 subjects, rolandic rhythm blocking in 53, GSRs in 95 , eye movements in 42 , changes in respiration rates in 63 , and an increase in muscle tension in only 1 subject. Apart from muscle tension, all components of the OR were present in only 11 subjects. If these measures are indicators of the OR and the OR is a unitary response, then it would be expected that the responses should occur together so that all subjects should 
show all components of the OR. Even if the OR was not elicited in all subjects because of inattention or for some other reason, more than $11 \%$ should show such a nexus. This failure and, in particular, the lack of concern shown by Sokolov for its implications in relation to the claimed unitary nature of the response must seriously weaken the validity of this concept. Surprisingly, no explicit investigation of the concept of a unitary OR has been undertaken in western psychophysiology, but some disturbing results have emerged almost incidentally in the recent literature. Before discussing these, it is necessary to briefly review present knowledge of the stimulusresponse relationships exhibited by individual physiological indicators commonly used in the OR field.

Graham and Clifton (1966) formalized the proposal that heart-rate (HR) deceleration best fitted the OR criteria of habituation and elicitation by low stimulus intensities. While results since then have not always consistently supported this view, it is generally accepted as correct by modern psychophysiology. Further, Jackson (1974) found that this HR deceleration followed Sokolov's predicted stimulus-intensity effect as outlined above, including the intensification at near-threshold stimulus intensities. This indicator of the OR is the only one to have been so confirmed in regard to these two aspects of its stimulus-response relationships.

Sokolov's statements regarding activity in the peripheral and cephalic vascular systems refer specifically to blood volume (BV) rather than to pulse volume (PV). BV represents the blood content of the system under consideration, while PV represents the variation in this produced by each pulse; PV thus bears a somewhat similar relation to BV as the GSR does to basal conductivity. Historically, PV has become the preferred measure in the West, and the design of recording instruments has developed accordingly. This has produced an additional complicating factor in this area, as the relationship between $\mathrm{PV}$ and $\mathrm{BV}$ has not been well established.

With peripheral pulse volume (PPV) and blood volume (PBV), there have been a number of useful studies relevant to this point. Davis, Buchwald, and Frankmann (1955) found a vasoconstriction in both measures which was directly related to stimulus intensity, but significant habituation failed to occur with stimulus repetition. Davis (1957) found a similar patterning of responses in both PPV and PBV measures with a wide variety of stimuli. Uno and Grings (1965) generally confirmed these results, and reported a correlation of 0.72 between the two measures, confirming their close relationship. Cohen and Johnson (1971), while reporting PPV response habituation (contrary to the above findings), noted that PBV results were so similar as to obviate the necessity of reporting PBV data. Levander, Lidberg, and Schalling (1974) also found that both PPV and PBV vasoconstrictive responses habituated, and recorded intraindividual correlations of the two measures ranging up to 0.98 . Ginsberg and Furedy (1974), on the other hand, found contradictory results, with PPV showing habituation while PBV did not. In spite of this, their reported correlation of 0.75 supports the general findings outlined above of a fairly close relation between the two measures of peripheral vascular activity, leading to the contention that they are alternate means of evaluating such activity. From the above reports, it must also be concluded that the question of habituation of this OR indicator has not been resolved. Earlier work relevant to this question and based on the use of PPV alone has been reviewed in Ginsberg and Furedy (1974) and leads to the same conclusion.

With regard to cephalic (C) vascular activity, a similar problem of CPV vs. CBV has existed, but there is a paucity of data even when compared with the peripheral vascular responses just discussed; the problem is further complicated by variation in recording site between experimenters. Cephalic vascular changes were of interest to Sokolov as they changed the blood supply to the brain, and he used the temporal area because "The data obtained from the temporal area were found to be closely comparable with those recorded directly from cerebral vessels in cases with skull defects" (Sokolov, 1963b, p. 39). Surface recording can only approximately reflect changes in the blood supply to the brain, and the accuracy of this approximation will depend on the relationship of the underlying vasculature to cerebral vessels. Sokolov indicated suitable areas as "temporal" (as above) and, in the same text, "the area of the bifurcation of the temporal and frontal arteries" (p. 38) and, using a pneumatic system, "the external auditory meatus" (p. 37). All these are closely related in terms of their blood supply. The frontal artery leaves the superficial temporal artery just above the ear, and both contribute to the blood supply of the temporal area. The branches of the superficial temporal artery anastomose with branches of the internal maxillary and carotid arteries, resulting in the observed correspondence of vascular change noted by Sokolov. The external auditory meatus is surrounded by and fed from branches of the internal maxillary artery and thus also reflects cerebral vascular changes. With the modern photoelectric pulse pick-ups available, the best recording site is probably where the superficial temporal artery crosses the zygoma, since it is there covered only by skin. No extensive data using this site is available. Most western work has involved recording from the forehead, but it might be expected that this choice of site could be unwise since the forehead is fed only by terminal branches of relatively small arteries 
which are relatively far removed from the cerebral vasculature.

Using this forehead site, Raskin, Kotses, and Bever $(1969 a$, b) looked at beat-by-beat CBV and CPV responses elicited by white noise signals of various intensities. The CBV response consisted of a dilation which discriminated stimulus intensity and habituated with stimulus repetition. In contrast, the CPV response was one of constriction which was not significantly affected by stimulus intensity but which did habituate. Such a finding posed problems for those who would replicate Sokolov's CBV results by the use of $\mathrm{CPV}$, since the two appeared to be different. However, Raskin et al. measured CPV only for the first 12 beats poststimulus, and found that the vasoconstriction peaked at beat 5, after which the CPV appeared to return to its base level. Keefe and Johnson (1970) extended their measurement of CPV to 23 beats poststimulus and found that tonal stimuli of moderate intensity elicited a biphasic response with vasoconstriction peaking at pulse 5 (as reported by Raskin et al.) followed by a vasodilation peaking at from 9 to 13 beats poststimulus. No significant habituation of this response pattern was obtained. Thus it appears that vasodilation can be found in both $C P V$ and CVB measures, allowing compatibility with the nature of the response predicted by Sokolov. Cohen and Johnson (1971) investigated CPV and CBV measures in response to tones of moderate intensity, and found that CPV and CBV results to be comparable, with biphasic responses in both systems. The later dilative components showed significant habituation with stimulus repetition.

This brief survey reveals some of the problems in this area, with CPV and CBV not clearly established as interchangeable indices of cephalic vascular activity. Stimulus-intensity effects and habituation of the vasodilation responses have not been clearly replicated, and the situation is further confused by the problems of recording site introduced above. Overall, there is little relevant information of any stature in this area of OR work.

The last index of the OR to be considered in detail here is respiration, which has had very little ORoriented investigation in recent years. Davis (1957) and Davis et al. (1955) reported a delay or pause followed by an increase in amplitude and a decrease in frequency of the respiratory cycle following simple visual and auditory stimulation; these changes appeared to increase with stimulus intensity. Johnson and Lubin (1967) confirmed the increase in duration of the respiratory cycle at the onset of low-intensity auditory stimulation. On the other hand, Smith and Strawbridge (1969) found a mean increase in both rate and amplitude following moderate-intensity stimulation. Porges and Raskin (1969) measured mean frequency and amplitude in 10-sec intervals before and after stimulation and found a small increase in frequency and a decrease in amplitude; these did not change with repeated stimulation. It must be noted, however, that their 10 -sec interval measures could mask small short-latency effects. McCallum, Burch, and Roessler (1969) found that both respiratory rate and amplitude (taken from four poststimulus cycles) showed an increase following visual and auditory stimulation, although this effect failed to reach significance for auditory stimulation. These changes increased directly with stimulus intensity. It appears, however, that the respiratory cycle(s) containing the stimulus onset and offset were omitted from the analysis, which would allow short-latency changes to occur without notice. It must be remembered that the duration of the respiratory cycle is of the order of $4 \mathrm{sec}$ in normal, relaxed subjects, and that such an ignoring of any cycle could result in the loss of valuable data.

An interesting application of the respiratory response produced by auditory stimulation is in the field of audiology. Rousey, Snyder, and Rousey (1964) tested the hypothesis that threshold tonal stimuli would produce the greatest disruption of the respiratory cycle. The assumption that near-threshold tones were associated with a slowing of the breathing rate was attributed to Woodworth (1938): "Sudden stimuli will make the subject 'catch his breath.' If he is listening to a faint sound, arrested breathing eliminates disturbing respiratory sound"' (p. 260). A respiration-determined threshold was defined as that intensity which produced the greatest increase in the respiratory cycle which contained the stimulus onset. These were within $20 \mathrm{~dB}$ of the conventional threshold for 7 out of 10 subjects. This was refined by Poole, Goetzinger, and Rousey (1966), who were able to use the respiratory response to estimate the auditory threshold to within $15 \mathrm{~dB}$ in 35 of $36 \mathrm{sub}$ jects. Overall, it is unfortunate that this technique has concentrated specifically on threshold effects rather than the magnitude of the increase in period as a function of intensity, but it must be conceded that the enhanced responding at threshold, as well as the nature of the response itself, supports Sokolov's predictions. It should also be pointed out that the measure of respiratory change used (i.e., increase in the period of the cycle containing the stimulus onset compared with prestimulus levels) appears to have captured a short-latency effect lost in most other studies discussed above. No systematic investigation of its relation to stimulus intensity or novelty has been attempted.

The literature relevant to GSR and EEG indicators of the OR has been recently reviewed by Barry $(1975,1976)$. It is generally accepted that the GSR both habituates and shows a linear dependence on stimulus intensity, while the magnitude of EEG 
alpha desynchronization habituates with stimulus repetition but shows no consistent stimulus-intensity effect. This difference in sensitivity to stimulusintensity variation with the GSR and EEG indices of the OR is indicative of the problems for Sokolov's unitary OR concept which are found in the literature. Some of these have emerged in the above survey of the results obtained with different physiological indices, and further studies which have investigated different indices together will now be considered briefly.

Uno and Grings (1965) found that HR changes exhibited a different stimulus intensity effect from the GSR or vascular responses, and that the GSR habituated clearly while the cardiovascular responses failed to do so. Raskin, Hattle, Harris, and De Young (1966) also found different habituation rates with $\mathrm{HR}$ and CBV. These differences were confirmed by Raskin, Kotses, and Bever (1969a) and extended to include different Stimulus Intensity by Repetition interactions, while Keefe and Johnson (1970) found that HR, PPV, and CPV all habituated differently. Jackson (1974) found different stimulus intensity effects at near-threshold intensities with GSR and HR deceleration; Coles, Sosdian, and Isaacson (1972) found that these two variables had different habituation rates under signal conditions.

Other evidence suggestive of problems in Sokolov's unitary OR concept comes from Holloway and Parsons (1971) and McDonald, Johnson, and Hord (1964). The former report established that while normal subjects showed habituation in HR, GSR, and EEG indices of the OR, brain-damaged subjects showed only GSR habituation; the latter report confirmed this with alert and drowsy subjects, as defined by EEG criteria. Cardiovascular indices (including PPV) failed to habituate in the drowsy group, while no difference was obtained with the GSR. These two reports suggest that a separate system is responsible for elicitation of the GSR, which is difficult to reconcile with Sokolov's position.

Thus differences have been reported between different $O R$ indices in regard to the magnitude of their stimulus intensity effects and habituation rates. Within the low-to-moderate range of intensity, where ORs are expected from Sokolov's theory to be reliably elicited, response magnitude is predicted to be linearly dependent on stimulus intensity except for enhancement at near-threshold levels. This intensity effect has been approximated by only GSR and HR deceleration. Respiratory pause appears to show a similar enhancement near threshold but has not been systematically investigated over an extensive intensity range. None of the other indices considered here have shown this low-intensity effect; rather, they appear to directly reflect stimulus intensity over a considerable range. Habituation is also an important criterion of the OR, but again, the response measures do not appear to consistently show this effect with stimulus repetition. The GSR and EEG indicators appear to habituate reliably, but HR deceleration can be said to do so only on the balance of experimental findings; significant numbers of studies have failed to observe habituation: Both PPV and CPV responses seem not to exhibit reliable habituation, and finally, respiratory pause has not been investigated sufficiently to allow firm conclusions to be drawn in this regard.

Finally, there is no evidence directly supporting Sokolov's concept of a unitary OR system. It is surprising that the work of Lacey and his colleagues, on tonic changes in the same physiological response systems, has not been taken as a warning by those working with the phasic OR concept: "There is nothing in the literature to support the statement that a correlation of +1.00 exists between measures of function of different autonomically-innervated structures. All published matrices of intercorrelations, indeed, show a large proportion of low and insignificant correlations. The most extensive modern studies of relationships among autonomic measures show that no single physiological measure can serve as an index to the state of other measures" (Lacey \& Lacey, 1958, p. 67). The lack of coherent behavior shown by the different indices as stimulus intensity and novelty are manipulated contradicts such a simplistic view of the physiological responsiveness of man as is implicit in Sokolov's conceptualization of the OR, but definitive data is lacking. The present investigation was directed to this lack of data in regard to simple or indifferent stimuli which have no significance for the organism apart from their physical parameters. To this end, no mention of the stimulus presentations was made to any subject so as to avoid attentional effects associated with experimenter demand.

\section{METHOD}

The subjects were 24 males between the ages of 18 and 25 years enrolled in an introductory psychology course at the University of Sydney who volunteered for the experiment to fulfill a course requirement. Stimuli consisted of $2-\mathrm{sec} 1,000-\mathrm{Hz}$ tones presented binaurally via earphones with an interstimulus interval randomly varying between 20 and $100 \mathrm{msec}$, with a mean of $60 \mathrm{sec}$. Each subject was given a unique cycle of the stimulus intensities of $20,30,40$, and $50 \mathrm{~dB}$ SPL (one of the 4 ! possible orders of 4 intensities) so that individual differences were confounded with stimulus order. Eight cycles of the four stimuli were presented to enable habituation to be studied. This procedure essentially replicated that used by Sokolov (1963b).

The EKG was recorded via a standard electrode placement (EKG lead 1) and recorded on a Grass IIID polygraph with standard EKG time constant and paper speed $15 \mathrm{~mm} / \mathrm{sec}$. PPV was recorded from a Physiograph photoelectric pulse pickup attached to the distal volar surface of the third finger of the subject's left hand (which rested on the arm of the subject chair). The PPV was written out on an E \& M Physiograph Six with a paper speed of $2.5 \mathrm{~mm} / \mathrm{sec}$. Another Physiograph photoelectric pulse pickup was placed against the zygoma, where a pulse could 
be manually felt. It was held in position by the earphones, and its output was recorded as the CPV on the Physiograph Six. Respiration was also recorded on the Physiograph Six from an $\mathrm{E} \& \mathrm{M}$ bellows pneumograph attached around the subject's chest. The electroculargram (EOG) was recorded on the Grass polygraph and used to check that the subject's eyes were open as instructed; it will not be discussed further. The GSR and three channels of EEG were also recorded. Results relevant to these indicators have been reported in Barry (1975) and Barry (1976), respectively, in relation to other aspects of Sokolov's OR theory and will not be discussed in detail here. Stimulus events were simultaneously indicated on a separate channel of each recording machine through the stimulus-presentation system.

Subjects were informed that they were involved in a correlational study involving the temporal covariation of various physiological indices. The headphones were supposedly used to mask noises from the elevator servicing the building. A pilot study had shown that this subterfuge was plausible, with some subjects helpfully reporting that they could still hear some elevator noises. Subjects sat in the dark with eyes open, in a comfortable armchair in a sound-reduced light-tight electrically shielded room. They were asked to remain still but awake; the eyes-open condition was designed to assist this last request.

\section{RESULTS}

The results of the experiment are presented below for each physiological indicator in turn.

\section{HR}

While the weight of evidence reviewed above supports an initial deceleration as the OR indicator, such a response has not been universally reported. It was therefore felt necessary to investigate the nature of the $H R$ response obtained here. The R-R interval containing the stimulus onset was arbitrarily defined as the first "poststimulus" interval, since it may have been perturbed by stimulus onset. R-R intervals were handscored from each record for three prestimulus and nine poststimulus intervals for the first stimulus cycle. Each of these periods was converted to a rate measure which was then used to derive the mean HRs for each pre- and poststimulus interval in the stimulus cycle by averaging over subjects and stimulus intensities. This response is shown in Figure 1 and labeled as the "standard" response. This form of response presentation is essentially the limit reached in previous studies, although it has the advantage of being based on the individual time unit of the R-R interval instead of (say) the 1-sec intervals commonly used as the time base in cardiotachometers. Averaging of activity in such natural time periods would appear to be more satisfactory than depending on some arbitrary time interval not intrinsically related to biorhythms.

This HR averaged within $R-R$ intervals shows a maximum change in the second and third poststimulus beats, but there is nothing in the literature to support this response being conceptualized as having a fixed latency. In fact, this averaged response is comparable with what would be obtained if a set of GSR protocols had the resistance scored at regular

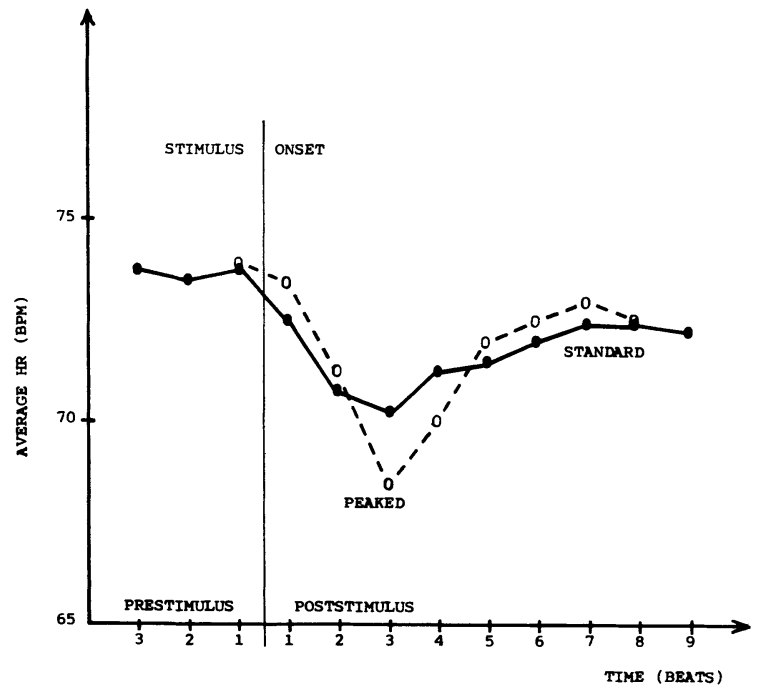

Figure 1. Mean HR response for the first stimulus cycle, showing both "standard" and latency-corrected ("peaked") curves.

short intervals and averaged for each interval over protocols. The resulting response would presumably show a drop in resistance following stimulus onset, but the nature of this response would differ from the responses used to obtain the compositedifferences in response latency would obscure the form of the averaged response. Accordingly, the record of beat-by-beat HRs from the first cycle of the individual protocols was scanned for such a deceleratory response at each stimulus presentation. The lowest HR occurring in the first five poststimulus beats was taken as the maximum response level. The means of these were obtained for all stimulus presentations in the first stimulus cycle. With reference to this "peaked" response, the average HRs for each of the three preceding and five following beats were also obtained. The resultant "peaked" response is also shown in Figure 1 and represents the average HR response corrected for different response latencies. It is to be noted that a pure deceleration of short latency (less than three HR periods), and of considerable magnitude for such low-intensity stimuli, has been found. This generally confirms the work of Graham and her associates on the nature of the HR OR.

The HR response was thus defined as the maximum deceleration relative to prestimulus HR occurring in the first five poststimulus beats. This was handscored off the individual protocols and converted to a percentage decrease in HR from Prestimulus Beat 1. Mean HR responses are shown as a function of intensity in Figure 3 and of stimulus repetition in Figure 4. These values were subjected to a two-way repeated measures trend analysis. No significant stimulus intensity, repetition effects or interactions were found, indicating that HR decelera- 


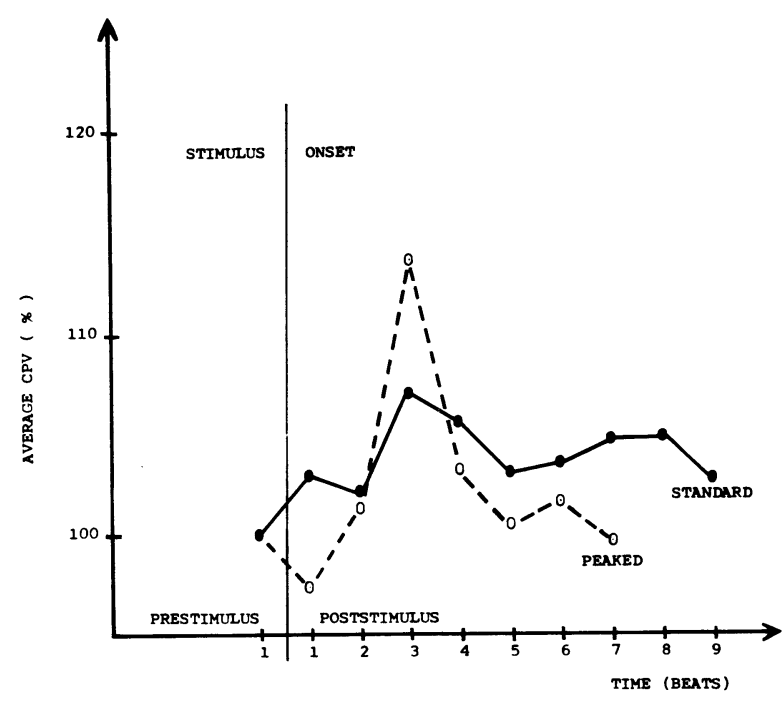

Figure 2. Mean CPV response for the first stimulus cycle, showing both "standard" and "peaked" curves.

tion failed to habituate or discriminate stimulus intensity.

\section{PPV}

Since it is well established that peripheral vasoconstriction reliably occurs in response to simple stimuli, the nature of this response per se was not investigated. The smallest pulse amplitude occurring between 7 and 12 beats poststimulus was taken as the point of maximum response. This was converted to a percentage decrease in PPV by comparison with the amplitude of prestimulus beat 1 . Mean values of this response are displayed as functions of intensity and stimulus cycle repetition in Figures 3 and 4, respectively. A two-way repeated measures trend analysis confirmed that there was no significant repetition effect-i.e., the PPV measures does not habituate. There was a significant linear relationship between PPV constriction and stimulus intensity $[F(1,69)=16.0, p<.001]$, but no significant interaction between stimulus intensity and repetition.

\section{CPV}

Since the form of the CPV response from the temporal region to stimuli of moderate intensity was not well established, a procedure similar to that used with the HR response was carried out. The amplitude of each pulse was handscored off the record for one prestimulus and nine poststimulus beats for the first stimulus cycle. These amplitudes were converted to percentages of the prestimulus amplitude and averaged over subjects and intensities within each beat to yield the "standard" response form shown in Figure 2. This diagram indicates that cephalic vasodilation occurs in response to simple stimuli, but it could be expected that the nature of this response is obscured by differences in response latency. Following the procedure used with HR, the maximum pulse amplitude occurring within the first six poststimulus beats was designated the maximum response. Relative to this pulse, the amplitudes of the three preceding and four following pulses were averaged over subjects and intensities to yield the "peaked" response shown also in Figure 2. the simple monophasic dilation confirms the proposal of Sokolov (1963b) and represents the first illustration of such a response free of latency confusions.

This change in CPV was used to define the CPV response as the maximum change in amplitude relative to the prestimulus pulse amplitude occurring within the first six poststimulus beats. Mean values

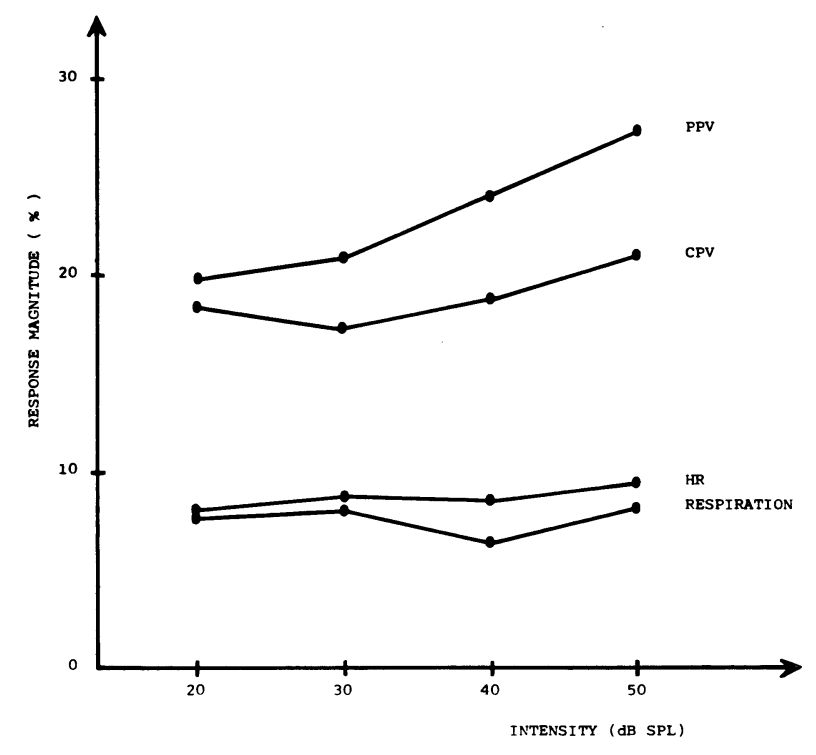

Figure 3. Response magnitude as a function of stimulus intensity.

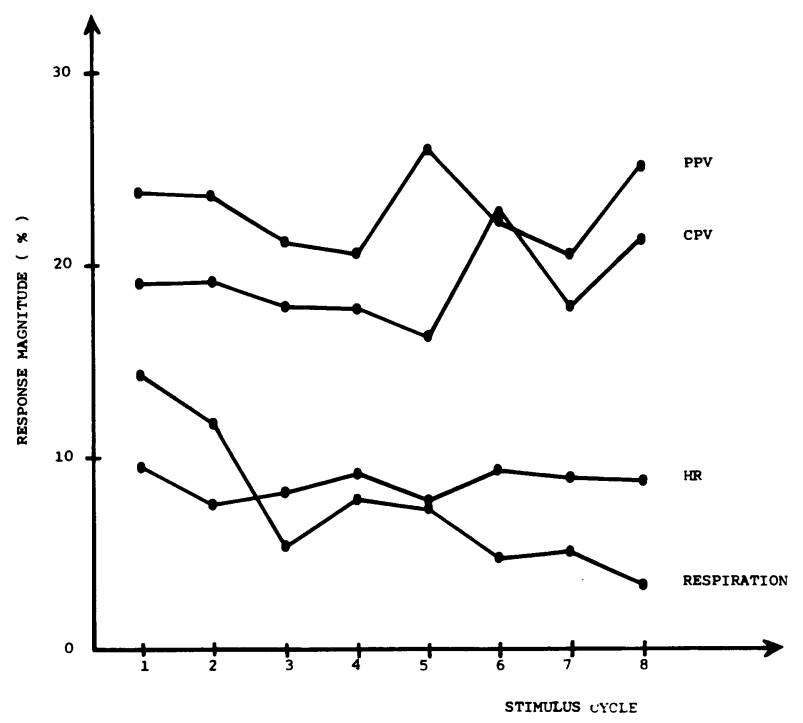

Figure 4. Response magnitude as a function of repeated stimulus cycles. 
of this response are displayed against stimulus intensity in Figure 3 and against repeated stimulus cycles in Figure 4. These values were submitted to a two-way repeated measures ANOVA which indicated no significant trends of response magnitude over stimulus intensity or repeated cycles, or Intensity by Repetition effects.

\section{Respiration}

For each stimulus presentation, the period of the respiratory cycle (measured from the onset of inspiration) immediately before stimulation and the cycle containing the stimulus onset were scored. The percentage increase in respiratory period at stimulus onset was taken as the measure of the respiratory index of the OR. Mean values of this response are shown as a function of intensity in Figure 3 and of stimulus repetition in Figure 4. A twoway repeated measures trend analysis found no significant intensity effect, but there was a significant habituation effect represented by a linear trend over stimulus cycles $[\mathrm{F}(1,23)=22.8, \mathrm{p}<.001]$. There were no significant interactions between stimulus intensity and repetition.

\section{DISCUSSION}

The results obtained with the three cardiovascular measures differ from the expectations derived from OR theory in their failure to habituate with stimulus repetition. It is to be remembered that HR deceleration and CPV dilation were clearly established in this study as the only response type consistently found in the protocols for these variables on the first stimulus cycle, and as such are the only possible candidates for the $O R$ label in these response systems. The failure of these measures to habituate is thus not simply an artifact of the response measures chosen, and it must be accepted that the response in the HR and CPV systems did not behave as predicted by Sokolov. The existing literature is minimal in regard to the CPV dilation, but it should be acknowledged that the failure of the HR response to habituate is contrary to the consensus of the experimental literature. This could be due to the failure of some earlier experimenters to consider the problem of response latencies: If latencies become more variable as the experiment proceeds (this might be a function of the state of alertness of the subject), then it could be expected that habituation of the averaged response as usually reported would appear to occur. Whether this artifactual habituation has been at the basis of previous findings, or whether the present results have some other cause, remains an open question. The failure of the PPV constrictive response to habituate is in keeping with the majority of previous reports; there is nothing here to allow speculation as to the different effects operat-
Table 1

Summary of Findings

\begin{tabular}{lcc}
\hline Varia & Intensity Effect & Repetition Effect \\
\hline HR & none & none \\
PPV & linear & none \\
CPV & none & none \\
Respiration & none & habituates \\
GSR & linear & habituates \\
EEG & complex & habituates \\
\hline
\end{tabular}

ing in those scattered experiments which have reported habituation in this measure.

The different stimulus intensity effects found here with these nonhabituating response measures also pose problems. The lack of sensitivity of the HR measure is certainly not as expected from the literature, which has reported both near-threshold enhancement and high-intensity DR competition so that a marked responsivity of this measure to variation in stimulus intensity was virtually assumed. There is no explanation presently available for these findings. The linear trend of response magnitude over stimulus intensity for the PPV measure is generally as expected from the literature, and there is insufficient data relevant to the CPV response available to result in firm predictions, so that the insensitivity of this measure to stimulus intensity is not controversial. It is apparent from these stimulusintensity effects that the cardiovascular responses have some independence and do not function as a single system.

In contrast to the cardiovascular responses, the respiratory response was found to habituate with stimulus repetition as predicted by Sokolov. Unfortunately, this response failed to show a significant stimulus intensity effect. While this was not as expected from the threshold work of Rousey and colleagues, insufficient parametric data have been available with which to compare these results, and they must stand alone at present.

It has previously been reported in relation to other aspects of Sokolov's OR theory that the GSR obtained in this study exhibited a linear stimulus intensity effect and habituated with stimulus repetition (Barry, 1975) while EEG alpha desynchronisation exhibited a complex nonlinear stimulus intensity effect and also habituated (Barry, 1976).

These different effects are summarized in Table 1. When these variations in stimulus intensity discrimination and habituation rates are considered, it is apparent that major problems exist in these data for Sokolov's unitary OR concept. Only the GSR has been found to respond to these independent variables as required of OR indices; the others fail to satisfy one or both of these criterial relationships. Further, not more than two of the six measures used covary even to the extent of having even similar relationships with these two variables. 
This study set out to investigate Sokolov's model OR elicited by indifferent stimuli. In obtaining, for the first time, parametric data relevant to six commonly used response measures in the same group of subjects, it appears that there is little to support such a unitary response system, since the six response measures investigated exhibit five different patterns of trend over the two variables of intensity and stimulus repetition. It would thus appear that the simple OR concept which has been widely accepted in western psychophysiology may be an extreme oversimplification, having little basis in experimental findings.

\section{REFERENCES}

BARRY, R. J. Low-intensity auditory stimulation and the GSR orienting response. Physiological Psychology, 1975, 3, 98-100.

BARRY, R. J. Failure to find the "local" EEG OR to low-level auditory stimulation. Physiological Psychology, 1976, 4, 171-174.

Cohen, M. J., \& Johnson, H. J. Effects of intensity and the signal value of stimuli on the orienting and defensive responses. Journal of Experimental Psychology, 1971, 88, 286-288.

Coles, M. G. H., Sosdian, B. J., \& IsaAcson, I. J. Heart rate and skin conductance responses to signal and nonsignal stimuli. Psychonomic Science, 1972, 29, 23-24.

DAvis, R. C. Response patterns. Transactions of the New York Academy of Science, 1957, 19, 731-739.

Davis, R. C., Buchwald, A. M., \& Frankmann, R. W. Autonomic and muscular responses and their relation to simple stimuli. Psychological Monographs, 1955, 69, No. 20. (71 pages)

Ginsberg, S., \& Furedy, J. J. Stimulus repetition, change, and assessments of sensitivities of and relationships among an electrodermal and two plethysmographic components of the orienting reaction. Psychophysiology, 1974, 11, 35-43.

Graham, F. K., \& Clifton, R. K. Heartrate change as a component of the orienting response. Psychological Bulletin, 1966, 65, 305-320.

Holloway, F. A., \& Parsons, O. A. Habituation of the orienting reflex in brain damaged patients. Psychophysiology, 1971, 8, 623-634.

JACKsON, J. C. Amplitude and habituation of the orienting reflex as a function of stimulus intensity. Psychophysiology. $1974,11,647-659$.

Johnson, L. C., \& Lubin, A. The orienting reflex during waking and sleeping. Electroencephalography and Clinical Neurophysiology, 1967, 22, 11-21.

KeEfe, F. B., \& Johnson, L. C. Cardiovascular responses to auditory stimuli. Psychonomic Science, 1970. 19, 335-337.
LACEY, J. I., \& LACEY, B. C. Verification and extension of the principle of autonomic response stereotypy. American Journal of Psychology, 1958, 71, 50-73.

Levander, S. E., Lidberg, L., \& Schalling, D. Habituation of the digital vasoconstrictive orienting response. Journal of Experimental Psychology, 1974, 102, 700-705.

McCollum, M., Burch, N. R., \& Roessler, R. Personality and respiratory responses to sound and light. Psychophysiology, 1969, 6, 291-300.

McDonald, D. G., Johnson, L. C., \& HoRd, D. J. Habituation of the orienting response in alert and drowsy subjects. Psychophysiology, 1964, 1, 163-173.

Poole, R., Goetzinger, C. P., \& Rousey, C. A study of the effects of auditory stimulation on respiration. Acta Otolaryngologica, 1966, 61, 143-152.

Porges, S. W., \& RAskin, D. C. Respiratory and heart rate components of attention. Journal of Experimental Psychology, 1969, 81, 497-503.

Raskin, D. C., Hattle, M., Harris, L., \& de Young, G. The effects of stimulus intensity, duration, and interstimulus interval on evocation and habituation of orienting and defensive reflexes. Psychophysiology, 1968, 4, 504-505.

Raskin, D. C., Kotses, H., \& BeVER, J. Autonomic indicators of orienting and defensive reflexes. Journal of Experimental Psychology, 1969, 80, 423-433.

Raskin, D. C., Kotses, H., \& Bever, J. Cephalic vasomotor and heart rate measures of orienting and defensive reflexes. Psychophysiology, 1969, 6, 149-159.

Rousey, C., SNYDER, C., \& Rousey, C. Changes in respiration as a function of auditory stimuli. Journal of Auditory Research, 1964, 4, 107-114.

Smith, D. B. D., \& Strawbridge, P. J. HR response to a brief auditory and visual stimulus. Psychophysiology, 1969, 6, 317-329.

Sokolov, E. N. The higher nervous activity and the problem of perception. Acta Psychologia, 1955, 13, 134-135.

Soxolov, E. N. Neuronal models and the orienting reflex. In M. A. Brazier (Ed.), The central nervous system and behaviour. Transactions of the 3rd conference. New York: Macy, 1960.

Sokolov, E. N. Higher nervous functions: The OR. Annual Review of Physiology, 1963, 25, 545-580. (a)

Sokolov, E. N. Perception and the conditioned reflex. Oxford: Pergamon Press, 1963. (b)

UNO, T., \& GRINGs, W. W. Autonomic components of orienting behavior. Psychophysiology, 1965, 1, 311-324.

Voronin, L. G., \& Sokolov, E. N. Cortical mechanisms of the orienting reflex and its relation to the conditioned reflex. In H. H. Jasper \& G. D. Smirnov (Eds.), The Moscow Colloquium on EEG and Clinical Neurophysiology. Electroencephalography and Clinical Neurophysiology, Suppl. 13, 1960.

WOODWORTH, R. Experimental psychology, New York: Holt, 1938.

(Received for publication August 2, 1976; revision accepted November $19,1976$. 